\title{
The Impact of Media on Public Health Awareness Concerning the Use of Natural Remedies Against the COVID-19 Outbreak in Saudi Arabia
}

\author{
Amna Alotiby 1 \\ Department of Hematology and \\ Immunology, Faculty of Medicine, Umm \\ Alqura University, Makkah, Saudi Arabia
}

Background: The coronavirus disease (COVID-19) pandemic has caused major health system problems and has fueled the emergence of various misinformation regarding preventive measures. The current study was conducted to evaluate the effect of media on raising the level of health awareness of Saudi Arabian populations regarding the medical misinformation about the use of natural remedies against COVID-19.

Methods: This descriptive cross-sectional study was conducted anonymously in the Kingdom of Saudi Arabia between May 1st and June 30, 2020. The sample size was calculated using version-3.0 of OpenEpi. An 11-item questionnaire was designed to assess the effect of media on raising the level of health awareness of the population regarding the medical misinformation about the use of natural remedies during this pandemic.

Results: Among 1300 participants, nearly half (55.4\%) reported having watched the Saudi Ministry of Health's (MOH) broadcasted awareness videos. Social media (78.9\%) was the main media platform on which they accessed these videos, followed by television $(14.9 \%)$, and the Internet (6.2\%). The MOH's awareness videos had a positive effect on $69.5 \%$ of participants, who reported a change in their attitudes toward natural remedies and discontinuing the use of them as preventive medicine. The most discontinued natural remedies after watching the awareness videos were gargling with salt water $(32.1 \%)$, followed by myrrh $(17.6 \%)$, and garlic $(12.9 \%)$. Positive effects were greater among participants with a low than high education level.

Conclusion: The findings of the current study indicate that the Saudi MOH's usage of social media channels has had a positive effect on the level of public health awareness concerning the reliability of natural remedies used during the pandemic. However, more research is needed to validate how social media networks can be used in a cross-cultural context to enhance health awareness and adaptation to healthy behaviors.

Keywords: health awareness, healthy behaviors, herbal preventive measures, Saudi Ministry of Health, MOH, coronavirus disease

\section{Introduction}

Recently, the outbreak of COVID-19 has had a devastating effect on the world. ${ }^{1}$ In Saudi Arabia, the first case of COVID-19 was identified in March 2020 and it began to spread rapidly after that. Consequently, the Saudi government introduced a range of steps to prevent the rapid spread of the virus, which included restrictions on the transportation and educational sectors. To reduce the spread of the virus, the entire population of Saudi Arabia was subsequently placed under a general curfew.
Correspondence: Amna Alotiby Department of Hematology and Immunology, Faculty of Medicine, Umm Alqura University, Makkah, Saudi Arabia Email aamogaty@uqu.edu.sa 
However, the spread of the disease could not be contained and the number of cases rose drastically to over 145,000 by the 18 th of June. ${ }^{2,3}$ While no specific vaccine had been invented against COVID-19 during the early phase of the outbreak, it was reported that multiple treatment regimens prescribed under different circumstances could control the severity and mortality rates of the virus to a degree with some side effects, although additional evidence was needed. $^{4}$

Products derived from natural sources have traditionally been considered the only treatment option for various diseases. To strengthen the immune system, natural products are widely used without a prescription. ${ }^{5}$ However, the herbal-drug interaction is a major disadvantage of using herbal products. ${ }^{6}$ Recently, medical misinformation on the effectiveness of natural remedies such as pepper, chili, black seeds, honey, onion, and garlic, etc. as preventive treatment has been spreading at an alarming rate on social media. ${ }^{7,8}$ Additionally, there has also been other misinformation on questionable treatment methods like killing the virus by gargling with salty water or myrrh to prevent entry into the lung. The spread of such misinformation can have disastrous consequences. In Nigeria, health officials discovered many cases of chloroquine overdose following the spread of misinformation in the media about its efficacy as a treatment for COVID-19. ${ }^{9}$ Without the proper understanding or awareness, most populations can be easily misled by this misinformation, which is unfortunate because studies have shown that natural remedies can help promote or maintain good individual health if taken properly but they cannot cure the disease or prevent infection. . $^{7,10,11}$ Furthermore, misinformation might also reduce the validity of new scientific discoveries regarding the treatment or vaccination of this disease. ${ }^{12}$

To the best of author's knowledge, there is no evidence supporting the effectiveness of natural remedies as either a safeguard against the virus or a cure for it. The World Health Organization (WHO) has also confirmed the lack of evidence on this aspect. ${ }^{13}$ Unfortunately, about $80 \%$ of the population in developed countries still believe in natural remedies without any regulation. ${ }^{14}$ It is recommended that policymakers include additional educational initiatives that improve public awareness of preventive measures and route disease transmission. Additionally, to ensure patient safety, the use of natural products must be evidencebased. ${ }^{2}$
The increase of social media usage during the pandemic has turned it into a source of information concerning COVID-19. Chan et al and Saud et al have especially highlighted the importance of social media as a viable tool for rapid knowledge dissemination during the early phase of COVID-19. ${ }^{15,16}$ To combat the misinformation, the Saudi Ministry of Health (MOH) has started broadcasting awareness videos through social media platforms like Facebook, Twitter, and Instagram. Additionally, the $\mathrm{MOH}$ has also initiated awareness programs to educate the public on not just the virus, but also proper ways of washing hands and wearing masks, the importance of curfew, and how the aforementioned measures and social distancing can help control the rate of viral transmission. ${ }^{17}$ Furthermore, the MOH has also issued awareness guidelines in different languages to raise awareness of both citizen and non-citizen residents about the virus, the necessary precautions against the virus, and the consequences of not complying with instructions. In addition to Arabic, the guidelines were written in English, Filipino, Portuguese, Russian, French, and Urdu. These guidelines were published through the $\mathrm{MOH}$ website, the official social media accounts of the government, and sent via text messages to everyone in Saudi Arabia. ${ }^{18}$

Research on the impact of social media on the level of health awareness regarding COVID-19 medical misinformation in the Saudi Arabian region remains scarce. Hence, this current study aims to fill that gap by evaluating the influence of social media platforms on the level of health awareness amongst the population in terms of medical misinformation about the use of natural remedies against the COVID-19 infection. Furthermore, to report the participant's behavior changes toward using natural remedies as protective measures by estimating the most common natural remedies stopped used after watching the awareness videos associated with age, gender, and educational level.

\section{Methodology}

\section{Study Design and Population}

An online descriptive cross-sectional study was conducted anonymously in the Kingdom of Saudi Arabia between the 1st of May and the 30th of June 2020. This study was approved by the Biomedical Ethics Committee of the Faculty of Medicine, Umm Alqura University, and was conducted in accordance with the Declaration of Helsinki. Every one of Saudi Arabia's general population (GP) aged 
18 years and above who watched the awareness videos broadcasted by Saudi's MOH were included in the study. The participants were excluded if they were younger than 18 or they had not watched the awareness videos. We had intended to perform a community-based national sampling survey, but it was not feasible due to the ongoing pandemic and the need for social distancing. Therefore, the data was collected via an online Google survey, which included an online informed consent form on the first page. It was also made clear that participation in this study was entirely voluntary. Participants were then asked to complete the questionnaire.

\section{Sample Size Calculation}

The sample size was calculated using version 3.0 of OpenEpi, taking into account the population size of Saudi Arabian adults (18 years and above), which is approximately 34 million. ${ }^{19}$ The minimum required sample size to achieve a $95 \%$ confidence interval and a $5 \%$ margin of error was 369 participants. About 1300 participants were enrolled in the current study; however, only 692 samples met the inclusion criteria of the study and were included in the analysis.

\section{Study Tool}

To assess the effect of media on raising the level of health awareness in the population regarding the medical misinformation about the use of natural remedies against COVID-19 infection, an 11-item questionnaire was developed. The format and content of the questionnaire were reviewed by four experts in the field of medical immunology, family medicine, preventive medicine, and clinical nutrition respectively to evaluate the appropriateness, relevance, clarity, and adequacy of the questions. The questionnaire was designed in English and translated into Arabic (the native language of the participants). The final Arabic questionnaire was pre-tested by the four experts (native Arabic speakers) and 10 volunteers from the general population. The final questionnaire was modified based on the feedback.

The questionnaire was divided into two sections. The first section included five questions that were used to evaluate the effect of the media on raising the level of health awareness in Saudi society during the COVID-19 pandemic. The first question asked the participants if they have watched the awareness videos correcting the misinformation about using natural remedies (herbs and food supplements) as preventive medicine. If they answered yes, they would proceed with the next question. If their answers were no, they were excluded from the study. The next two questions asked them about the sources of these videos and how they accessed them. The fourth question asked them how these videos affected the level of their awareness by changing their beliefs and trust in the use of natural remedies to fight COVID-19. The final question in this section was to evaluate the behavioral changes of the participants who watched the awareness videos by asking them which of the 14 listed items (most common herbs and protective measures) that were circulated during the outbreak on social media had they stopped using. ${ }^{20}$ The second section consisted of six questions about the participants' general information, such as the area of residence, nationality, gender, age, educational level, and health status.

\section{Statistical Analysis}

The obtained data was filtered and put through the IBM SPSS version 20 (SPSS, Inc. Chicago, IL, USA). Percentage analysis and chi-square test were carried out. Statistical significance was considered at a $p$ value less than 0.05 .

\section{Results}

\section{Characteristics of the Study Participants}

A total of 692 people answered "Yes" to the first question. The demographic characteristics of the patients are summarized in Table 1 . Nearly half $(47.4 \%)$ of the participants were from the Western region, followed by nearly onethird $(30.1 \%)$ from the central region, and $8.8 \%$ from the eastern region. The majority $(95.7 \%)$ of the participants were Saudi, with most of them being women (76.6\%). More than half $(58.4 \%)$ of the participants were young adults (18-40 years), while more than one-third $(37.6 \%)$ were middle-aged adults (41-60 years). Approximately $83.1 \%$ of the participants were university graduates holding a bachelor's or postgraduate degree. Approximately $31.2 \%$ of the participants had existing chronic health problems.

\section{Awareness}

Table 2 presents the percentages of participants who have watched the awareness videos, sources of information, and the perceived effects of watching the awareness. Nearly half of the participants $(55.4 \%)$ reported having watched an awareness video. Social media was the main channel 
Table I Demographic Data of Study Participants Who Have Seen the Awareness Videos, Saudi Arabia $(n=692)$

\begin{tabular}{|l|l|l|}
\hline Demographic Variable & No & $\%$ \\
\hline Region & & \\
Central region & 208 & $30.1 \%$ \\
Eastern region & 61 & $8.8 \%$ \\
North region & 52 & $7.5 \%$ \\
Southern region & 43 & $6.2 \%$ \\
Western region & 328 & $47.4 \%$ \\
\hline Nationality & & \\
Saudi & 662 & $95.7 \%$ \\
Non-Saudi & 30 & $4.3 \%$ \\
\hline Gender & & \\
Male & 162 & $23.4 \%$ \\
Female & 530 & $76.6 \%$ \\
\hline Age group & & \\
Young adults & 404 & $58.4 \%$ \\
Middle-aged adults & 260 & $37.6 \%$ \\
Older adults & 28 & $4.0 \%$ \\
\hline Educational level & & \\
Less than high school & 16 & $2.3 \%$ \\
High school & 101 & $14.6 \%$ \\
Bachelor's degree & 396 & $57.2 \%$ \\
Post graduate degree & 179 & \\
\hline Chronic disease history & 216 & \\
Yes & 476 & \\
No & & \\
\hline
\end{tabular}

through which they watched the videos $(78.9 \%)$, followed by television (14.9\%), and the Internet $(6.2 \%)$. In terms of the awareness videos' sources, Saudi $\mathrm{MOH}$ was the most reported $(71.5 \%)$, followed by the WHO (13.6\%). In terms of the degree of effect the videos had on the participants, $69.5 \%$ of respondents reported it having a profound effect, $24.4 \%$ having some effect, and the remaining $6.1 \%$ having no effect.

Figure 1 displays the herbs and natural products that were stopped due to the effect of watching the awareness videos as reported by the participants. The most frequently used natural products were salt water (32.1\%), Myrrh $(17.6 \%)$, garlic $(12.9 \%)$, black seeds $(11 \%)$, chili pepper $(10.7 \%)$, honey $(9.7 \%)$, and lemon $(9.1 \%)$. The least reported were ginger $(5.7 \%)$, banana $(3.5 \%)$, and olive oil $(2.8 \%)$.

Table 3 presents the association between the effect of awareness videos on raising the level of awareness about natural remedies used during the COVID-19 pandemic and the demographic variables. The survey shows that the videos had a higher effect on participants from the Central region than from the Eastern region, with statistical significance $(p=0.044)$. The positive effects were greater among participants who had a low education level (below high school) than among those with a postgraduate degree $(p=0.015)$. However, no significant differences were observed between gender (male vs female),

Table 2 Frequency of Watching Awareness Videos to Correct for the Medical Information of Herbs Role During COVID-19 Pandemic, Its Source, and Effect $(n=692)$

\begin{tabular}{|c|c|c|}
\hline Awareness Video Data & No & $\%$ \\
\hline \multicolumn{3}{|l|}{ Watching awareness videos } \\
\hline Yes $(n=692)$ & 692 & $55.4 \%$ \\
\hline No $(n=608)$ & 557 & $44.6 \%$ \\
\hline \multicolumn{3}{|l|}{ Media platforms (Source of information) } \\
\hline Social media (WhatsApp, Twitter, Snapchat, and Instagram) & 546 & $78.9 \%$ \\
\hline Television & 103 & $14.9 \%$ \\
\hline Internet (Websites) & 43 & $6.2 \%$ \\
\hline \multicolumn{3}{|l|}{ The source for the awareness videos } \\
\hline Saudi Ministry of Health $(\mathrm{MOH})$ & 495 & $71.5 \%$ \\
\hline WHO in the Middle East & 94 & $13.6 \%$ \\
\hline Do not know what the source is & 103 & $14.9 \%$ \\
\hline \multicolumn{3}{|c|}{$\begin{array}{l}\text { The effect of watching awareness videos on participants' beliefs and changing the use of natural remedies as } \\
\text { protective measures (Stopping their use to fight COVID-19 infection). }\end{array}$} \\
\hline Yes & 481 & $69.5 \%$ \\
\hline To some extent & 169 & $24.4 \%$ \\
\hline No effect & 42 & $6.1 \%$ \\
\hline
\end{tabular}




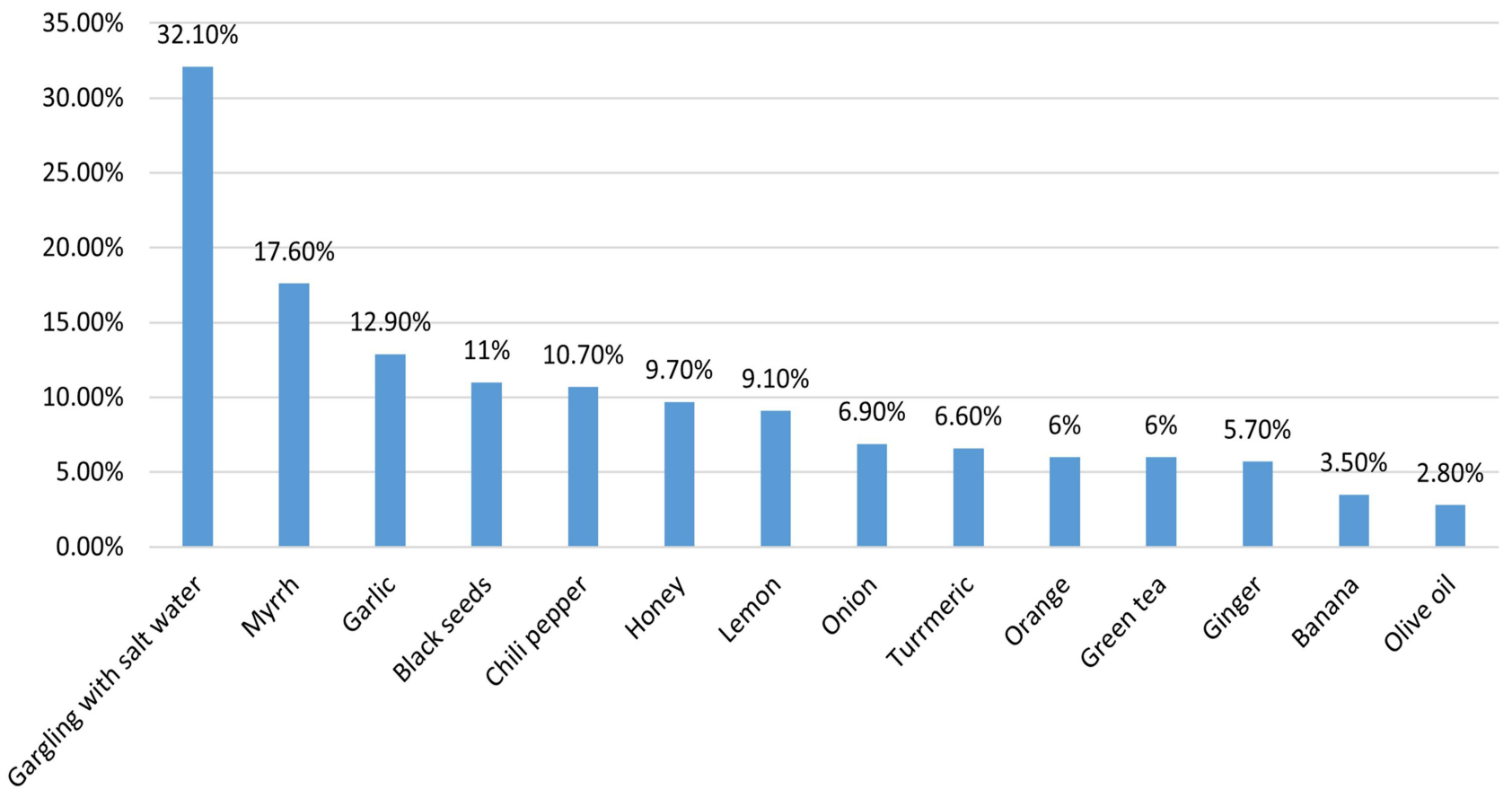

Figure I Herbs and natural products that were stopped used after watching the awareness videos.

age groups, and among participants with chronic health problems.

\section{Discussion}

The outbreak of COVID-19 was declared a public health emergency in January 2020 by WHO and was named a pandemic in March 2020. Sadly, due to the lack of treatment options for COVID-19, many people have turned to alternative solutions, including the use of herbal extracts and natural products to improve their immunity and reduce the risk of infection, which has not proven to be effective. Building public awareness is thus crucial to the efforts of controlling the spread of COVID- $19 .{ }^{21}$ Hence, the main focus of this study was to evaluate the effect of the Saudi MOH's awareness videos broadcasted via social and mass media on raising the level of health awareness regarding the medical misinformation of natural remedies against COVID-19.

In this study, most of the participants were from the western and central regions. Our findings show that social media was the main source of information, which confirms what Alyami et al reported - that the biggest motivators for participants to pursue herbal products were social media and the Internet. ${ }^{2}$ Alanezi et al also observed in their study that most of their participants favored getting news updates from press releases and online government websites over social media sites. ${ }^{22}$ Similarly, most of the videos watched by our participants were sourced from the Saudi Ministry of Health (71.5\%), followed by the WHO $(13.6 \%)$. These findings corroborated the findings of Kwok et al, who reported that most participants relied more on the Saudi Ministry of Health than on recognized bodies such as the WHO and healthcare experts on the subject of Covid-19. ${ }^{23}$ This can be explained by the Saudi people's previous positive experience with the $\mathrm{MOH}$ during the Middle East Respiratory Syndrome (MERS) outbreak in $2012 .{ }^{24}$ In terms of the impact the videos had on the use of herbs or natural products during the COVID-19 pandemic, more than $75 \%$ of the respondents reported a significant effect. This could be because the use of herbal products has become common among patients, including in their struggle with infectious diseases such as MERS and SARS, as Hwang et al reported, thus encouraging more researchers to document herbal products' use. ${ }^{25}$

After watching the awareness videos, there was a change in the behavior of participants concerning the use of natural remedies as protective measures. Frequent use of natural remedies was discontinued, particularly the use of saltwater, Myrrh, and garlic. This indicates that the Saudi MOH's use of social media to broadcast awareness videos has had a positive effect on the population's beliefs and practices regarding the use of natural remedies as protective measures. This result 
Table 3 Association Between Demographic and Effect of Watching Awareness Videos on Changing the Use of Herbs or the Natural Products During the COVID-19 Pandemic

\begin{tabular}{|c|c|c|c|c|c|c|c|}
\hline \multirow[t]{3}{*}{ Demographic Data } & \multicolumn{6}{|c|}{$\begin{array}{l}\text { Effect of Watching Awareness Videos on Changing the Use of Herbs or the Natural } \\
\text { Products During the COVID-19 Pandemic }\end{array}$} & \multirow[t]{3}{*}{ P-value } \\
\hline & \multicolumn{2}{|c|}{ No Effect } & \multicolumn{2}{|c|}{$\begin{array}{l}\text { To Some Extent (Moderate } \\
\text { Effect) }\end{array}$} & \multicolumn{2}{|c|}{ Yes (High Effect) } & \\
\hline & No & $\%$ & No & $\%$ & No & $\%$ & \\
\hline Region & & & & & & & $0.044 *$ \\
\hline Central region & 10 & $4.8 \%$ & 57 & $27.4 \%$ & $14 \mid$ & $67.8 \%$ & \\
\hline Eastern region & 8 & $13.1 \%$ & 17 & $27.9 \%$ & 36 & $59.0 \%$ & \\
\hline North region & I & $1.9 \%$ & 19 & $36.5 \%$ & 32 & $61.5 \%$ & \\
\hline Southern region & 3 & $7.0 \%$ & 9 & $20.9 \%$ & 31 & $72.1 \%$ & \\
\hline Western region & 20 & $6.1 \%$ & 67 & $20.4 \%$ & 241 & $73.5 \%$ & \\
\hline Nationality & & & & & & & 0.983 \\
\hline Saudi & 40 & $6.0 \%$ & 162 & $24.5 \%$ & 460 & $69.5 \%$ & \\
\hline Non-Saudi & 2 & $6.7 \%$ & 7 & $23.3 \%$ & 21 & $70.0 \%$ & \\
\hline Gender & & & & & & & 0.093 \\
\hline Male & 9 & $5.6 \%$ & 50 & $30.9 \%$ & 103 & $63.6 \%$ & \\
\hline Female & 33 & $6.2 \%$ & 119 & $22.5 \%$ & 378 & $71.3 \%$ & \\
\hline Age group & & & & & & & 0.425 \\
\hline Young adults & 24 & $5.9 \%$ & 90 & $22.3 \%$ & 290 & $71.8 \%$ & \\
\hline Middle aged adults & 16 & $6.2 \%$ & 69 & $26.5 \%$ & 175 & $67.3 \%$ & \\
\hline Older adults & 2 & $7.1 \%$ & 10 & $35.7 \%$ & 16 & $57.1 \%$ & \\
\hline Educational level & & & & & & & $0.015^{*}$ \\
\hline Less than high school & 0 & $0.0 \%$ & 0 & $0.0 \%$ & 16 & $100.0 \%$ & \\
\hline High school & 6 & $5.9 \%$ & 24 & $23.8 \%$ & 71 & $70.3 \%$ & \\
\hline Bachelor's degree & 23 & $5.8 \%$ & 87 & $22.0 \%$ & 286 & $72.2 \%$ & \\
\hline Post graduate degree & 13 & $7.3 \%$ & 58 & $32.4 \%$ & 108 & $60.3 \%$ & \\
\hline Chronic disease history & & & & & & & 0.916 \\
\hline Yes & 14 & $6.5 \%$ & 54 & $25.0 \%$ & 148 & $68.5 \%$ & \\
\hline No & 28 & $5.9 \%$ & 115 & $24.2 \%$ & 333 & $70.0 \%$ & \\
\hline
\end{tabular}

Notes: * Pis significant at $<0.05$.

corroborates the findings of a previous study analyzing the impact of social media platforms on public health protection during the COVID-19 pandemic. The previous study reported an overall positive impact on public health protection against COVID-19 among those who acquired accurate pandemicrelated information over social media. ${ }^{26}$

The effect of the videos on the use of ginger, banana, and olive oil was minimal, as they were the least discontinued natural remedies used as protective measures. This indicates that the population still has relatively high faith in the use of ginger and its ability to improve the immunity of individuals. This supports the findings of previous studies by
Alyami et al and Alotiby et al which also reported the high popularity of ginger, onion, and garlic in the general population as a way of improving immunity and reducing the risk of developing COVID-19., ${ }^{2,20}$ Regarding banana and olive oil, the prevalence of their use by the general population was very low in comparison to other natural remedies at the outset. ${ }^{20}$ This explains why they were the least discontinued natural remedies. Per the survey, positive effects of the broadcasted awareness videos on changing the participants' beliefs and trust toward the use of natural remedies to fight COVID-19 infection were reported more in participants from the Central than from the Eastern region $(p=0.044)$. 
This might be due to the population diversity in the Central region of Saudi Arabia. Riyadh, the capital city of Saudi Arabia, is located here. Most people living in Riyadh moved there from different Saudi regions for either work or studies. $^{27}$ These positive effects were also greater among participants with a low education level $(p=0.015)$ than those who had a post-graduate degree. These findings were in accordance with a previous study by Alyami et al, which showed that participants with higher levels of education had better knowledge about using natural remedies as preventive measures against COVID-19. ${ }^{2}$ In addition, other researchers have also shown that people with a higher level of education have a better understanding about the preventive steps of various diseases. ${ }^{28,29}$

\section{Conclusion}

Improving the awareness of the targeted groups, particularly the low-educated ones, is critical to handling the COVID-19 threat. A lack of awareness can result in the worsening of the situation. Awareness campaigns using accurate information from credible sources can remedy the issue of misinformation. These campaigns should also be active in improving public attitudes towards effective preventive measures. The findings of the present study have also shown that the use of social media channels may positively influence public health awareness and inspire positive behavioral changes. Thus, we believe that public health authorities should use social media platforms as an effective tool to increase public health awareness through the dissemination of informative videos to targeted populations. However, more research is needed to validate how social media networks can be used in a cross-cultural context to enhance health awareness and adaptation to healthy behaviors. There are also some limitations to our current study. Our ability to define causality among study variables was limited by the cross-sectional survey design of the study. An online questionnaire is an important limitation of this study, which limited our accessibility to a larger sample size due to the lockdown situation. The generalization of the current study result is also potentially restricted since the study was limited to people who had access to the online questionnaire.

\section{Data Sharing Statement}

The original contributions presented in the study are included in the article; further inquiries can be directed to the corresponding author.

\section{Ethics Approval and Informed Consent}

The current study was approved by the biomedical ethics committee of the Faculty of Medicine, Umm Alqura University, and that it was conducted in accordance with the Declaration of Helsinki. The data were collected via an online Google survey, which included an online informed consent form on the first page, and participation in this study was voluntary. Once the participants choose to participate in the study after reading the informed consent on the survey's front page, they are processed to complete the questionnaires.

\section{Acknowledgments}

The author is grateful to the deanship of scientific research at Umm Al-Qura University, Makkah, Saudi Arabia, for the continuous support.

\section{Author Contributions}

Amna Abdullah Alotiby is the sole author of this article. The author made a substantial contribution to conception and design, acquisition of data, or analysis and interpretation of data; took part in drafting the article or revising it critically for important intellectual content; agreed to submit to the current journal; gave final approval of the version to be published; and agree to be accountable for all aspects of the work.

\section{Funding}

The author declares that no funds were received to conduct this research.

\section{Disclosure}

The author declares no conflicts of interest for this work and that the research was conducted in the absence of any commercial or financial relationships that could be construed as a potential conflict of interest.

\section{References}

1. Tripathi R, Alqahtani SS, Albarraq AA, et al. Awareness and preparedness of COVID-19 outbreak among healthcare workers and other residents of South-West Saudi Arabia: a cross-sectional survey. Front Public Health. 2020;8:482. doi:10.3389/fpubh.2020.00482

2. Alyami HS, Orabi MAA, Aldhabbah FM, et al. Knowledge about COVID-19 and beliefs about and use of herbal products during the COVID-19 pandemic: a cross-sectional study in Saudi Arabia. Saudi Pharm J. 2020;28(11):1326-1332. doi:10.1016/j.jsps.2020.08.023

3. Saudi Ministry of Health. COVID-19. Available from: https://www. moh.gov.sa/en/CCC/events/COVID-19/Pages/2020.aspx. Accessed December 12, 2021. 
4. Taccone FS, Gorham J, Vincent J-L. Hydroxychloroquine in the management of critically ill patients with COVID-19: the need for an evidence base. Lancet Respir Med. 2020;8(6):539-541. doi:10.1016/s2213-2600(20)30172-7

5. Martini M, Gazzaniga V, Bragazzi NL, Barberis I. The Spanish influenza pandemic: a lesson from history 100 years after 1918. J Prev Med Hyg. 2019;60(1):E64-E67. doi:10.15167/2421-4248/ jpmh2019.60.1.1205

6. Posadzki P, Watson L, Ernst E. Herb-drug interactions: an overview of systematic reviews. Br J Clin Pharmacol. 2013;75(3):603-618. doi:10.1111/j.1365-2125.2012.04350.x

7. Ang L, Lee HW, Choi JY, Zhang J, Soo lee M. Herbal medicine and pattern identification for treating COVID-19: a rapid review of guidelines. Integr Med Res. 2020;9(2):100407. doi:10.1016/j. imr.2020.100407

8. Lissiman E, Bhasale AL, Cohen M. Garlic for the common cold. Cochrane Database Syst Rev. 2014;11:CD006206. doi:10.1002/ 14651858.CD006206.pub4

9. Busari S, Adebayo B. Nigeria records chloroquine poisoning after trump endorses it for coronavirus treatment. CNN. Available from: https://edition.cnn.com/2020/03/23/africa/chloroquine-trump-nigeriaintl/index.html. Accessed December 20, 2020.

10. Al Akeel MM, Al Ghamdi WM, Al Habib S, Koshm M, Al Otaibi F. Herbal medicines: Saudi population knowledge, attitude, and practice at a glance. $J$ Family Med Prim Care. 2018;7(5):865-875. doi:10.4103/jfmpc.jfmpc_315_17

11. Mahish PK, Mahobia R, Yadav J. Use and awareness of herbal medicines among literate population. Int J Pharma Bio Sci. 2016;7 (4). doi:10.22376/ijpbs.2016.7.4.p174-178

12. Kasulis K. Patient 31, and South Korea's sudden spike in coronavirus cases. Al Jazeera News. 2020.

13. World Health Organization. Coronavirus disease (COVID-19) advice for the public: myth busters. Available from: https://www.who.int/ emergencies/diseases/novel-coronavirus-2019/advice-for-public /myth-busters. Accessed December 20, 2020.

14. Pathak K, Das R. Herbal medicine-a rational approach in health care system. Eur J Herb Med. 2013;86:86-89.

15. Chan AKM, Nickson CP, Rudolph JW, Lee A, Joynt GM. Social media for rapid knowledge dissemination: early experience from the COVID -19 pandemic. Anaesthesia. 2020;75(12):1579-1582. doi:10.1111/anae.15057

16. Saud M, Mashud M, Ida R. Usage of social media during the pandemic: seeking support and awareness about COVID-19 through social media platforms. J Public Aff. 2020;20(4):e2417. doi:10.1002/pa.2417

17. Saudi Ministry of Health. Awareness plateform-COVID19. Available from: https://www.moh.gov.sa/en/awarenessplateform/VariousTopics/ Pages/COIVD-19.aspx. Accessed May 25, 2021.

18. Saudi Ministry of Health. MOH issues COVID-19 awareness guidelines in many languages. Available from: https://www.moh.gov.sa/en/ Ministry/MediaCenter/News/Pages/News-2020-03-17-001.aspx. Accessed May 25, 2021.
19. General Authority for Statistics in Saudi Arabia. Population estimates. Available from: https://www.stats.gov.sa/en/43. Accessed October 5, 2020.

20. Alotiby A, Al-Harbi L. Prevalence of using herbs and natural products as a protective measure during the COVID-19 pandemic among the Saudi population: an online cross-sectional survey. Saudi Pharmaceutical Journal. 2021;29(5):410-417. doi:10.1016/j. jsps.2021.04.001

21. Tasnim S, Hossain MM, Mazumder H. Impact of rumors and misinformation on COVID-19 in social media. J Prev Med Public Health. 2020;53(3):171-174. doi:10.3961/jpmph.20.094

22. Alanezi F, Aljahdali A, Alyousef S, et al. Implications of public understanding of COVID-19 in Saudi Arabia for fostering effective communication through awareness framework. Front Public Health. 2020;8:494. doi:10.3389/fpubh.2020.00494

23. Kwok KO, Li KK, Chan HHH, et al. Community responses during early phase of COVID-19 epidemic, Hong Kong. Emerg Infect Dis. 2020;26(7):1575-1579. doi:10.3201/eid2607.200500

24. Aldarhami A, Bazaid AS, Althomali OW, Binsaleh NK. Public perceptions and commitment to social distancing "staying-at-home" during COVID-19 pandemic: a national survey in Saudi Arabia. Int J Gen Med. 2020;13:677-686. doi:10.2147/IJGM.S269716

25. Hwang JH, Cho HJ, Im HB, Jung YS, Choi SJ, Han D. Complementary and alternative medicine use among outpatients during the 2015 MERS outbreak in South Korea: a cross-sectional study. BMC Complement Altern Med. 2020;20(1):147. doi:10.1186/s12906020-02945-0

26. Al-Dmour H, Masa'deh R, Salman A, Abuhashesh M, Al-Dmour R. Influence of social media platforms on public health protection against the COVID-19 pandemic via the mediating effects of public health awareness and behavioral changes: integrated model. $J$ Med Internet Res. 2020;22(8):e19996. doi:10.2196/19996

27. Khraif RM, Salam AA, Nair PS, Elsegaey I. Migration in Saudi Arabia: Present and Prospects. Singapore: Springer; 2019:99-123. doi:10.1007/978-981-13-9224-5_5

28. Naser AY, Dahmash EZ, Alwafi H, et al. Knowledge and practices towards COVID-19 during its outbreak: a multinational cross-sectional study. medRxiv. 2020:20063560. doi:10.1101/ 2020.04.13.20063560

29. Zhong BL, Luo W, Li HM, et al. Knowledge, attitudes, and practices towards COVID-19 among Chinese residents during the rapid rise period of the COVID-19 outbreak: a quick online cross-sectional survey. Int $J$ Biol Sci. 2020;16(10):1745-1752. doi:10.7150/ ijbs. 45221
International Journal of General Medicine

\section{Publish your work in this journal}

The International Journal of General Medicine is an international, peer-reviewed open-access journal that focuses on general and internal medicine, pathogenesis, epidemiology, diagnosis, monitoring and treatment protocols. The journal is characterized by the rapid reporting of reviews, original research and clinical studies across all disease areas. The manuscript management system is completely online and includes a very quick and fair peer-review system, which is all easy to use. Visit http://www.dovepress.com/ testimonials.php to read real quotes from published authors. 